\title{
Complete algorithm for the calculation light patterns inside the ocular media
}

\author{
JORGE PÉREZ, DAVID MAS, CARLOS ILLUECA*, JUAN J. MIRET, \\ CARMEN VÁZQUEZ and CONSUELO HERNÁNDEZ \\ Departmento de Óptica, Universidad de Alicante, \\ PO Box 99, 03080, Alicante, Spain
}

(Received 6 July 2004; in final form 19 October 2004)

\begin{abstract}
Realistic mathematical models are of great importance for studying the optical performance of the human eye. Light propagation algorithms provide robust methods to calculate field distributions inside a homogeneous medium, and thus they can be applied to the study of light patterns inside the anterior and posterior chambers of the eye. Dealing with real eyes implies using very short propagation distances together with highly refractive power surfaces. Thus, the general solutions of the field equation are used instead of paraxial Fresnel solutions. Conditions of application and sampling conditions of the method are clearly stated here. Numerical evaluation of the different refractive surfaces is also analysed. The main result is that a complete algorithm to obtain light patterns at any axial distance inside the eye is proposed. The method uses real corneal measures and axial distances together with crystalline models. Statistical results and individual predictions show the validity of the model. Application of the method is illustrated with the study of a bifocal intraocular lens.
\end{abstract}

\section{Introduction}

Numerical analysis and simulation of light propagation through the human eye and interaction with its different optical surfaces have become a fundamental tool in understanding the optical performance of the human eye [1-6]. Applications derived from fundamental investigation of optical systems may have a real impact on the final quality of the life of subjects affected by severe refractive dysfunction.

The optical quality of the visual system is determined by the final image formed on the retina, which has been analysed in a wide variety of conditions (young and old people, aphakic and pseudoaphakic eyes, post-surgical eyes, etc.) and applying several objective methods that provide complementary results.

Most of the cited methods for studying the optical performance of the eye are based on two different methods. The first consists of analysing the wavefront aberration function $W(x, y)$. It is obtained through laser-ray-tracing techniques or Hartmann-Shack interferometers [7-10]. Both of these are implemented in commercial devices that are used in ophthalmological centres and allow measuring up to the seventh order of aberrations.

*Corresponding author. Email: illueca@ua.es 
Other methods that are commonly used are based on the point spread function (PSF) and the modulation transfer function (MTF) [11-13]. Both of these can be obtained by a double-pass device, which is also part of commercial sophisticated optical instrumentation that is used in ophthalmological clinics. The PSF and MTF allow determination of image quality and even reconstructing the final retinal image for complex scenes.

Besides the above mentioned functions, it is common to use single parameters that quantify the quality of an optical system. One of these is the Strehl ratio which can be derived from PSF measures. The Strehl ratio is the ratio of the maximum energy peak produced by an aberrated system to that produced by an aberration-free system, both calculated on a particular image plane. Other quality parameters have been analysed by papers in the references, and some of these have been proposed by us [14].

Additionally to experimental measures, realistic mathematical models are of great importance for studying the optical performance of the eye. Much surgery on the eye is not reversible. Thus, a priori knowledge of the problem is necessary. Precise models about the performance of the human eye under a wide variety of refractive circumstances may provide such information.

Usually, light propagation inside the eye has been studied by ray-tracing algorithms. These algorithms provide information about ray trajectory, but they only manage a few rays, compared with the number of curvature points that can be obtained with corneal topography.

Some of our previous papers have been devoted to analysing light propagation inside the eye. Several algorithms have been proposed and discussed, and interesting results have been obtained. In [6] we proposed the use of a near-field calculation algorithm for evaluating the image quality produced by an aphakic eye. The corneal phase transmittance is calculated from the data provided by a standard videokeratograph. Assuming that the incoming beam is known, we calculated the light distribution after passing the cornea. The propagation algorithm is then applied to this distribution and light patterns are obtained at the desired distance.

Applying the same method to a complete eye model requires the propagation of a convergent light pattern from the cornea to the crystalline lens. The product of the incoming distribution and the transmittance of the crystalline lens will be then propagated to the retina.

As has been demonstrated in [15], propagation of convergent beams requires additional analysis to that for free-space propagated beams. The convergence elements that deflect the beam interfere with the kernel of the propagation integral, and thus sampling relations must be analysed carefully.

Apart from propagation, a complete eye model must take into account two additional elements: the cornea and the crystalline lens. The cornea can be accessed without using any invasive technique. Modern videokeratographs provide accurate data of curvature that may be incorporated in a sophisticated eye model.

The crystalline lens cannot be accessed from outside and thus it can only be specified through indirect measures. Furthermore, this element is of a complex graded index structure that changes with age and with accommodation state. Measuring these characteristics require sophisticated set-ups that are not usual in ophthalmological clinics. This is the main reason why we have decided to use a mathematical model instead of direct experimental data. 
There are many models of crystalline lenses with several advantages [16]. Among them, we are interested in the model of Kasprzak [17]. The main advantages of this model are that it conveniently reproduces morphology, convergence and spherical aberration of the crystalline lens. Furthermore, it can be conveniently described by analytic equations. In order to adapt this model to the group under study, we have completed it with data from Koretz et al. [18], Brown [19] and Cook et al. [20] which permit convenient modifications of values of thickness and curvature according to the age of subjects.

With all these elements we propose a complete algorithm that permits calculation and visualization of light patterns at any propagated distance inside the eye. The objective of this paper is to define clearly the different steps of the algorithm and its conditions of application. With the algorithm we have obtained several quality parameters that are commonly used in the literature (the Strehl ratio, energy dispersion, etc.). We have compared our results with similar results obtained by other workers through experimental methods. We have also performed individual predictions for each subject. The results of all these parameters show that the method is comparable with other existing techniques and may complement their results.

In section 2, we shall present the algorithms for calculating the propagated field inside the ocular media where distances are very short. Because of this, known propagation algorithms are analysed and conveniently modified. In section 3, we review the optical surfaces of the eye: the cornea and the crystalline lens together with the particular considerations that we use in our eye model. In section 4, we present the complete eye method together with statistical results, and in section 5 we outline some applications of our algorithm. Finally, in section 6 we summarize the conclusions and results and also propose future development of this method.

\section{Propagation through the ocular media}

The beam propagation problem inside the eye is formulated as follows. Let us consider an isotropic, homogeneous and non-dispersive medium covering the halfspace $z \geqslant 0$. Let us also consider an entrance pupil of circular shape at the plane $z=0$ illuminated by a normally incident plane wave of wavelength $\lambda$. The generated optical field at a distance $z$, evaluated at the spatial coordinates $\left(x_{z}, y_{z}\right)$ can be described by a complex function $u_{z}$ given by [21]

$$
\begin{aligned}
u_{z}\left(x_{z}, y_{z}, z\right)= & \int_{-\infty}^{+\infty} \tilde{u}_{0}(\xi, \eta) \exp \left(\mathrm{i} \frac{2 \pi}{\lambda} z\left[1-(\lambda \xi)^{2}-(\lambda \eta)^{2}\right]^{1 / 2}\right) \\
& \times \exp \left[\mathrm{i} 2 \pi\left(\xi x_{z}+\eta y_{z}\right)\right] \mathrm{d} \xi \mathrm{d} \eta,
\end{aligned}
$$

where $\tilde{u}_{0}(\xi, \eta)$ is the Fourier transform of the field at $z=0$ evaluated at the spatial frequencies $(\xi, \eta)$. It is usually called the angular spectrum of the field. Note that equation (1) is an exact solution of the Helmholtz wave equation. Note also that this equation is symmetric with respect to both $x_{z}$ and $y_{z}$ and their respective conjugated variables $\xi$ and $\eta$. Therefore, from now on we shall use one-dimensional notation in order to facilitate the derivation of the formulas.

On the other hand, when we consider beam propagation under paraxial conditions, propagated light patterns can be adequately described by the standard 
Fresnel integral:

$$
u_{z}\left(x_{z}\right)=\exp \left(\mathrm{i} \frac{\pi}{\lambda z} x_{z}^{2}\right) \int_{-\infty}^{+\infty} u_{0}\left(x_{0}\right) \exp \left(\mathrm{i} \frac{\pi}{\lambda z} x_{0}^{2}\right) \exp \left(-\mathrm{i} \frac{2 \pi}{\lambda z} x_{z} x_{0}\right) \mathrm{d} x_{0},
$$

where $u_{0}\left(x_{0}\right)$ represents the spatial field distribution at $z=0$. In our particular case, the distance from the cornea to the crystalline lens is very short (about $4 \mathrm{~mm}$ ) and pupil apertures are of the same order. Thus we are not strictly under paraxial conditions and the Fresnel integral does not guarantee enough accuracy in light pattern calculations in the anterior chamber.

Equation (1), however, permits calculation of the field at any distance from the object. Proper discretization of this expression allows numerical evaluation of light patterns inside the eye, provided that the Nyquist conditions are fulfilled.

In the references we find a variety of numerical algorithms for calculation of diffracted light patterns. In [22-24], a discrete calculation method based on the angular spectrum propagation is analysed. Propagation calculation through those algorithms is accomplished by means of the Fourier transform of the Fresnel integral kernel, and thus they are valid only under paraxial conditions. Let us consider an input signal of size $\Delta x_{0}$ that has been discretized in $N$ samples. The propagated pattern, calculated through an angular spectrum propagation method provides

$$
\left(u_{z}\right)_{\mu} \propto \mathrm{DFT}^{-1}\left\{\exp \left(-\mathrm{i} \pi \frac{\lambda z}{\left(\Delta x_{0}\right)^{2}} \tilde{m}^{2}\right) \operatorname{DFT}\left[u_{0}\left(\frac{m \Delta x_{0}}{N}\right)\right\},\right.
$$

where $m, \tilde{m}$ and $\mu$ are discrete indices in space, Fourier and Fresnel domains respectively, DFT is discrete Fourier transform and $\Delta x_{0}$ is the size of the object support window. $\Delta x_{0}$ must not be confused with the size of the pupil aperture, which is taken to the smaller. This is because in discrete transformations it is usual to pad with zeros the input signal in order to avoid vignetting effects.

Discretization of equation (1) provides a propagation algorithm that is equivalent to this one. The only important difference is that the kernel that multiplies the Fourier transform of the signal is slightly modified, giving:

$$
\left(u_{z}\right)_{\tilde{m}}=\mathrm{DFT}^{-1}\left\{\exp \left[\mathrm{i} \frac{2 \pi}{\lambda} z\left(1-\lambda^{2} \frac{\tilde{m}^{2}}{\Delta x_{0}^{2}}\right)^{1 / 2}\right] \operatorname{DFT}\left[u_{0}\left(\frac{m \Delta x_{0}}{N}\right)\right]\right\} .
$$

Thus, the full algorithm consists of considering a plane wave that enters the eye. The beam is modified by the phase transmittance of the cornea. Propagation from the cornea to the crystalline lens is accomplished through the above expression. At this point, the effect of the phase transmittance of the crystalline lens must be considered. Application of equation (4) provides, finally, a light pattern at any distance from the lens.

Applicability of the algorithm is limited by aliasing in the kernel of propagation. Applying the Nyquist condition on this phase factor results in

$$
\frac{2 \pi}{\lambda} z\left|\left(1-\lambda^{2} \frac{N^{2}}{4\left(\Delta x_{0}\right)^{2}}\right)^{1 / 2}-\left(1-\lambda^{2} \frac{N^{2}-2 N+4}{4\left(\Delta x_{0}\right)^{2}}\right)^{1 / 2}\right| \leqslant \pi
$$


which implies that this calculation method is valid for distances fulfilling

$$
z \leqslant \frac{f_{1}}{2}\left[\left(1-\frac{\lambda N}{4 f_{1}}\right)^{1 / 2}+\left(1-\frac{\lambda N}{4 f_{1}}+\frac{\lambda}{f_{1}}\right)^{1 / 2}\right] \leqslant f_{1},
$$

where $f_{1}$ is given by

$$
f_{1}=\frac{\Delta x_{0}^{2}}{\lambda N} \geqslant \frac{\lambda N}{4}
$$

The inequality comes from considering that $z$ is a positive real number and thus the radicands are non-negative.

Despite the generality of this solution, some peculiarities of the optical system of the eye must be observed and incorporated in the above exposed conditions. One consideration is that the eye is a convergent system. The presence of a convergent phase factor affecting the object interferes with the kernel of propagation, and conditions of applicability of the method change. Let us consider the situation when the input pattern converges to a positive distance $z_{\mathrm{c}}$. Mathematically, we can extract the converging factor from the object, and thus make a more precise analysis of the situation, which is expressed as

$$
\left(u_{z}\right)_{\tilde{m}}=\mathrm{DFT}^{-1}\left\{\exp \left[\mathrm{i} \frac{2 \pi}{\lambda} z\left(1-\lambda^{2} \frac{\tilde{m}^{2}}{\Delta x_{0}^{2}}\right)^{1 / 2}\right] \operatorname{DFT}\left[u_{0}\left(\frac{m \Delta x_{0}}{N}\right) \exp \left(-\mathrm{i} \pi \frac{m^{2} \Delta x_{0}^{2}}{\lambda N^{2}} \frac{1}{z_{\mathrm{c}}}\right)\right]\right\}
$$

Now, the factor inside the direct DFT and the propagation kernel that multiplies it must be correctly sampled. Nyquist condition applied on the converging phase gives

$$
\frac{\Delta x_{0}^{2}}{\lambda N^{2}} \frac{1}{z_{\mathrm{c}}} \leqslant \frac{1}{N} \longrightarrow N \geqslant \frac{\Delta x_{0}^{2}}{\lambda z_{\mathrm{c}}} \longrightarrow f_{1} \leqslant z_{\mathrm{c}},
$$

which can be combined with condition, thus giving

$$
z \leqslant \frac{f_{1}}{2}\left[\left(1-\frac{\lambda N}{4 f_{1}}\right)+\left(1-\frac{\lambda N}{4 f_{1}}+\frac{\lambda}{f_{1}}\right)\right] \leqslant z_{\mathrm{c}} .
$$

This condition imposes serious restrictions on the distances at which the pattern can be calculated. Note that, beyond the convergence distance, the Nyquist criterion is no longer fulfilled and patterns are affected by aliasing.

In the case of the human eye, propagation must be accomplished through the anterior and the vitreous chambers and both propagations are affected by convergence. In the first propagation from the cornea to the lens, typical values that we have used are

$$
\Delta x_{0}=6 \mathrm{~mm}, \quad f_{1}=z_{\mathrm{c}}=31.7 \mathrm{~mm}, \quad \lambda_{0}=632.8 \mathrm{~nm}, \quad n=1.3375 .
$$

Note that $\lambda_{0}$ is the light wavelength at the vacuum. Inside the ocular media, this parameter must be corrected by the index of refraction $n$, that is $\lambda=\lambda_{0} / n$. With all these data we find that the number of samples needed is $N=2400$ and equation (8) is accurate up to a distance of $31.55 \mathrm{~mm}$. The distance from the cornea to the crystalline lens is about $4 \mathrm{~mm}$, and thus patterns in this region are correctly calculated. 
For the second propagation from the lens to the retina we have considered slightly different parameters. The pattern converges towards the retina at about $18 \mathrm{~mm}$ from the crystalline lens. Therefore, the new input parameters are

$$
\Delta x_{0}=6 \mathrm{~mm}, \quad f_{1}=z_{\mathrm{c}}=18 \mathrm{~mm}, \quad \lambda_{0}=632.8 \mathrm{~nm}, \quad n=1.336 .
$$

With these conditions we find that correct sampling of patterns from the lens to the retina is achieved for $N=4096$.

Note that different samplings are needed for the anterior and vitreous chambers respectively. This implies resampling at the crystalline lens plane. To our knowledge, resampling of amplitude is not especially problematic. On the other hand, phase variation is too fast at the borders, and interpolation of data introduces many artiefacts that distort the final image. Thus, we prefer to use the highest number of samples from the beginning of the calculation. The large number of samples may be a serious drawback when real-time applications are needed. Nevertheless, current computers are capable of managing a great number of data and calculate all matrix computations implied in the process in reasonable time (few minutes). Fortunately, forthcoming computers will reduce this computation time.

\section{Evaluation of the transmittance of the corneal and crystalline lens}

Apart from propagation through the ocular media, the optical transmittances of the cornea and crystalline lens must be also considered. These functions are obtained here by calculating the phase delay introduced by each element on the total field. To this end, we calculate the particular morphology of each element. The optical path between an input and output plane is evaluated at each point. Finally, this optical path difference is interpreted as a phase delay [25].

The procedure to calculate light patterns at any distance inside the eye is as follows: determination of corneal transmittance, propagation through the anterior chamber, determination of the crystalline lens transmittance and, again, propagation to the final image plane. In figure 1 we show a schematic eye with different parameters that we use in our calculation. The reference planes are denoted by $\Pi$, $\Sigma$ and $\Omega$ and the retinal plane. The distances $z_{\mathrm{AC}}$ and $z_{\mathrm{AL}}$ are the anterior chamber length and the total axial length respectively, while $2 d$ is the total thickness of the lens. We denote the refractive indexes of the aqueous humour, the lens and the vitreous humor by $n_{\mathrm{a}}, n_{\mathrm{c}}$ and $n_{\mathrm{v}}$ respectively. With all these data, let us analyse, separately, the field distribution at each reference plane.

\subsection{Passing the cornea}

Corneal morphology is reconstructed for each subject from topographic data. In this study, we have not considered the effect of the posterior surface of the cornea. This surface separates two media with similar refractive indices and contributes to a very small fraction of the total eye power. However, to consider the effect of both surfaces we have used an equivalent index $n=1.3375$ for the aqueous humour [26]. Procedures and algorithms are detailed in the references (see [6] for example). Let us consider the anterior chamber. Let $z$ be the sagittal depth of a surface point with coordinate $x$ and let $z_{\Pi}$ be the distance between the corneal apex and a reference plane $\Pi$ limited by the pupil radius $R_{\mathrm{p}}$. If we consider an incoming plane wave 


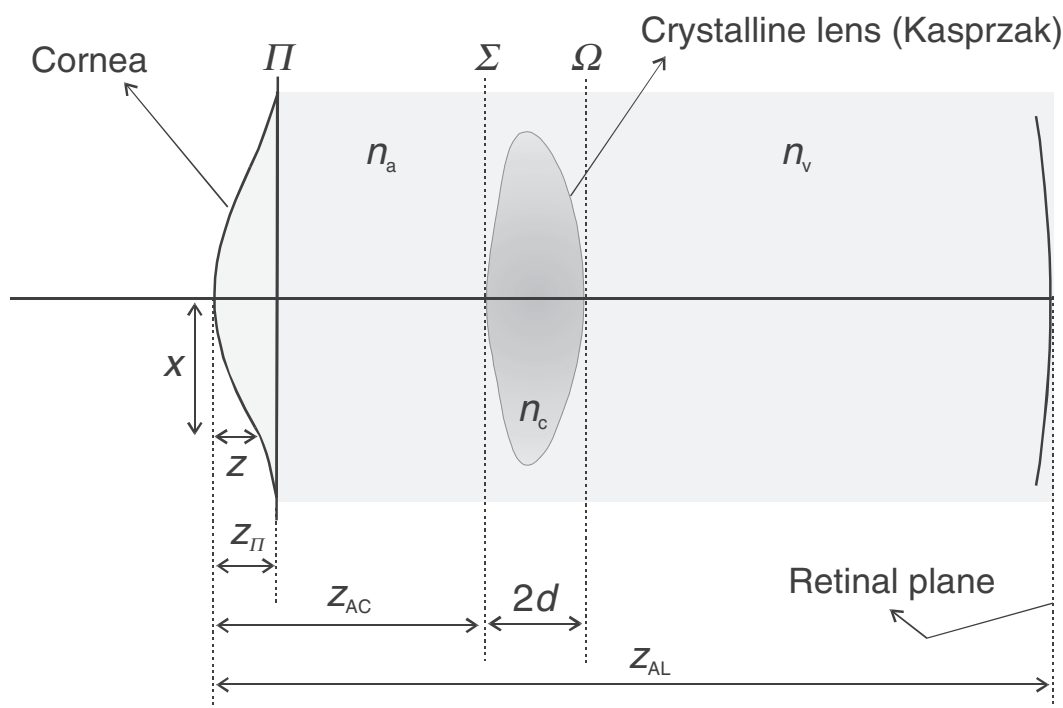

Figure 1. Schematic representation of the human eye. The crystalline lens has been represented according to the Kasprzak [17] model.

with wavelength $\lambda_{0}$, light distribution at the corneal output plane $\Pi$ can be expressed as

$$
u_{\Pi}\left(x_{\Pi}\right)=\exp \left\{\mathrm{i} \frac{2 \pi}{\lambda_{0}} z\left(x_{\Pi}\right)+n_{\mathrm{a}}\left[z_{\Pi}-z\left(x_{\Pi}\right)\right]\right\} .
$$

In order to propagate this distribution, it must be conveniently discretized, in $N$ samples, thus obtaining an output matrix $\left(u_{\Pi}\right)_{m}$ evaluated at $x=m \Delta x_{0} / N$.

\subsection{Propagation through the anterior chamber}

The anterior chamber length $z_{\mathrm{AC}}$ is determined for each subject with a non-contact biometer. As we pointed out before, a typical value of this parameter is about $4 \mathrm{~mm}$. Having in mind equation (4), the field distribution at the entrance plane $\Sigma$ of the lens is

$\left(u_{\Sigma}\right)_{\tilde{m}}=\mathrm{DFT}^{-1}\left(\exp \left\{\mathrm{i} \frac{2 \pi n_{\mathrm{a}}}{\lambda_{0}}\left(z_{\mathrm{AC}}-z_{\Pi}\right)\left[1-\left(\frac{\lambda_{0}}{n_{\mathrm{a}}}\right)^{2} \frac{\tilde{m}^{2}}{\left(\Delta x_{0}\right)^{2}}\right]^{1 / 2}\right\} \operatorname{DFT}\left[u_{\Pi}\left(\frac{m \Delta x_{0}}{N}\right)\right]\right)$.

\subsection{Passing the crystalline lens}

Actual crystalline lens models can take into account the particular morphology of the lens, different refractive index distribution models and even accommodation. Among the wide variety of models, we have selected here that proposed by Kasprzak [17]. In this model the lens has revolution symmetry and its anterior and posterior 
profiles are described by hyperbolic cosine functions modelled by hyperbolic tangents:

$$
\begin{aligned}
\rho(\varphi) & =\rho_{\mathrm{A}}(\varphi)+\rho_{\mathrm{P}}(\varphi)-d, \\
\rho_{\mathrm{A}}(\varphi) & =\frac{a_{\mathrm{A}}}{2}\left[\cosh (\pi-\varphi) b_{\mathrm{A}}-1\right]\left\{1-\tanh \left[m\left(s_{\mathrm{A}}-\varphi\right)\right]\right\}+d, \\
\rho_{\mathrm{P}}(\varphi) & =\frac{a_{\mathrm{P}}}{2}\left[\cosh \left(b_{\mathrm{P}} \varphi\right)-1\right]\left\{1-\tanh \left(m\left(s_{\mathrm{P}}-\varphi\right)\right)\right\}+d,
\end{aligned}
$$

$\rho_{\mathrm{A}}, \rho_{\mathrm{P}}$ and $\rho$ being the anterior, posterior and total profiles respectively in polar coordinates, and $d$ is half the total thickness of the lens (see figure 1). The coefficients $a_{\mathrm{A}, \mathrm{P}}$ and $b_{\mathrm{A}, \mathrm{P}}$ depend on the anterior and posterior axial curvature radii:

$$
\begin{aligned}
& a_{\mathrm{A}, \mathrm{P}}=\frac{d R_{\mathrm{A}, \mathrm{P}}\left(R_{\mathrm{A}, \mathrm{P}}-d\right) p_{\mathrm{A}, \mathrm{P}}}{3\left(R_{\mathrm{A}, \mathrm{P}}-d\right)\left(2 R_{\mathrm{A}, \mathrm{P}}-d\right)-R_{\mathrm{A}, \mathrm{P}}^{2}}, \\
& b_{\mathrm{A}, \mathrm{P}}=\left(\frac{d\left(R_{\mathrm{A}, \mathrm{P}}-d\right)}{R_{\mathrm{A}, \mathrm{P}} a_{\mathrm{A}, \mathrm{P}}}\right)^{1 / 2},
\end{aligned}
$$

and remaining parameters are dimensionless magnitudes that describe the exact form of the surface. In this paper we have used the following parameters proposed by Popiolek and Kasprzak [27] for the unaccommodated crystalline lens: $p_{\mathrm{A}}=0.929$, $p_{\mathrm{P}}=0.764, m=6.11, s_{\mathrm{A}}=1.62$ and $s_{\mathrm{P}}=1.65$.

Values for curvature radius and central thickness have been modified here by taking into account the age of the subject. Following indications given in [18-20], the final parameters that we have used are

$$
\begin{aligned}
d & =1.73+0.0065 \times \text { age } \\
R_{\mathrm{A}}(\mathrm{mm}) & =16.815-0.104 \times \text { age } \\
R_{\mathrm{P}}(\mathrm{mm}) & =8.719-0.015 \times \text { age }
\end{aligned}
$$

where age is expressed in years.

Returning to our propagation problem, the optical path that is covered by the light entering the lens at the plane $\Sigma$ and leaving it at the plane $\Omega$ is

$$
\begin{aligned}
\operatorname{cryst}(\varphi)= & n_{\mathrm{a}}[d-\rho(\pi-\varphi) \cos \varphi]+n_{\mathrm{c}}[\rho(\pi-\varphi)+\rho(\varphi)] \cos \varphi \\
& +n_{\mathrm{v}}[d-\rho(\varphi) \cos \varphi],
\end{aligned}
$$

which may be conveniently discretized. Finally, the light transmittance of the lens gives

$$
u_{\Omega}\left(x_{\Omega}\right)=u_{\Sigma}\left(x_{\Sigma}\right) \exp \left(\mathrm{i} \frac{2 \pi n_{\mathrm{a}}}{\lambda_{0}} \operatorname{cryst}\left(x_{\Sigma}\right)\right)
$$

\subsection{Propagation through the posterior chamber}

The final step of the algorithm consists of propagating the distribution in equation (19) up to the plane of interest. If one is interested in the retinal plane, its position can be determined by a non-contact biometer. As we pointed out before, a typical distance from the lens to the retina is about $18 \mathrm{~mm}$. In any case, the final distribution 
situated at a distance $z$ from the corneal apex is

$\left(u_{z}\right)_{\tilde{m}}=\mathrm{DFT}^{-1}\left(\exp \left\{\mathrm{i} \frac{2 \pi n_{\mathrm{v}}}{\lambda_{0}}\left(z-2 d-z_{\mathrm{AC}}\right)\left[1-\left(\frac{\lambda_{0}}{n_{\mathrm{v}}}\right)^{2} \frac{\tilde{m}^{2}}{\left(\Delta x_{0}\right)^{2}}\right]^{1 / 2}\right\} \operatorname{DFT}\left[u_{\Omega}\left(\frac{m \Delta x_{0}}{N}\right)\right]\right)$.

From this expression, one may calculate any function defined on an image plane, such as the PSF and the MTF or even other quality parameters [28].

\section{Results}

We have applied the algorithm to analyse the image formation process on real subjects. We have studied our model on 44 healthy eyes. The group is composed of people with ages ranging from 21 to 43 years with an average age of 31 years and a standard deviation of \pm 9 years. The selection criteria were as follows: a manifest refractive sphere of -2.0 to +2.0 dioptres, and a cylinder of less than 1 dioptre; no corneal surgery, no contact lens user and no missing data points within the $6.0 \mathrm{~mm}$ central zone on Humphrey Atlas corneal topographic maps (Carl Zeiss, Inc.). Refractive measurements have been made with a refractive precision of 0.25 diaptres and angular precision of $5^{\circ}$. Before taking part in the study all subjects provided informed consent in accordance with the Declaration of Helsinki.

For each subject we have obtained the corneal topography data with a previously calibrated Atlas Humphrey corneal topography system. The reproducibility of the measures was checked by measuring the radius and corneal asphericity several times, obtaining similar results. We also took biometric measures of the anterior chamber and axial length with a Zeiss intracular lens (IOL) master non-contact biometer, with a precision of $0.01 \mathrm{~mm}$. All lengths were measured three times with a dispersion error less than $0.02 \mathrm{~mm}$.

We have calculated the theoretical MTF for all the subjects in the image plane with the highest Strehl ratio. As we shall explain below, this plane does not always coincide with the retinal plane measured with the biometer.

With these MTFs, we have reproduced the typical form of the curve for normal population. In figure 2 we show a comparison between the radial average MTF for all our subjects, obtained through this method and the MTF obtained by other workers through double-pass techniques [29-33]. All curves here use $\lambda=633 \mathrm{~nm}$ except that from the work of Guirao et al. [33] which uses $\lambda=543 \mathrm{~nm}$, and thus the curve is slightly higher. We can see that agreement between all results is good, with differences between the functions being less than $5 \%$ in all measured frequencies.

The accuracy of MTFs obtained with our algorithm has also been checked by adjusting our curves to analytical expressions of the MTF proposed by several workers. In particular, we have compared the average MTF obtained for all the subjects with the expression

$$
\operatorname{MTF}(f)=A \exp \left(-\frac{f}{B}\right)+(1-A) \exp \left(-\frac{f}{C}\right)
$$



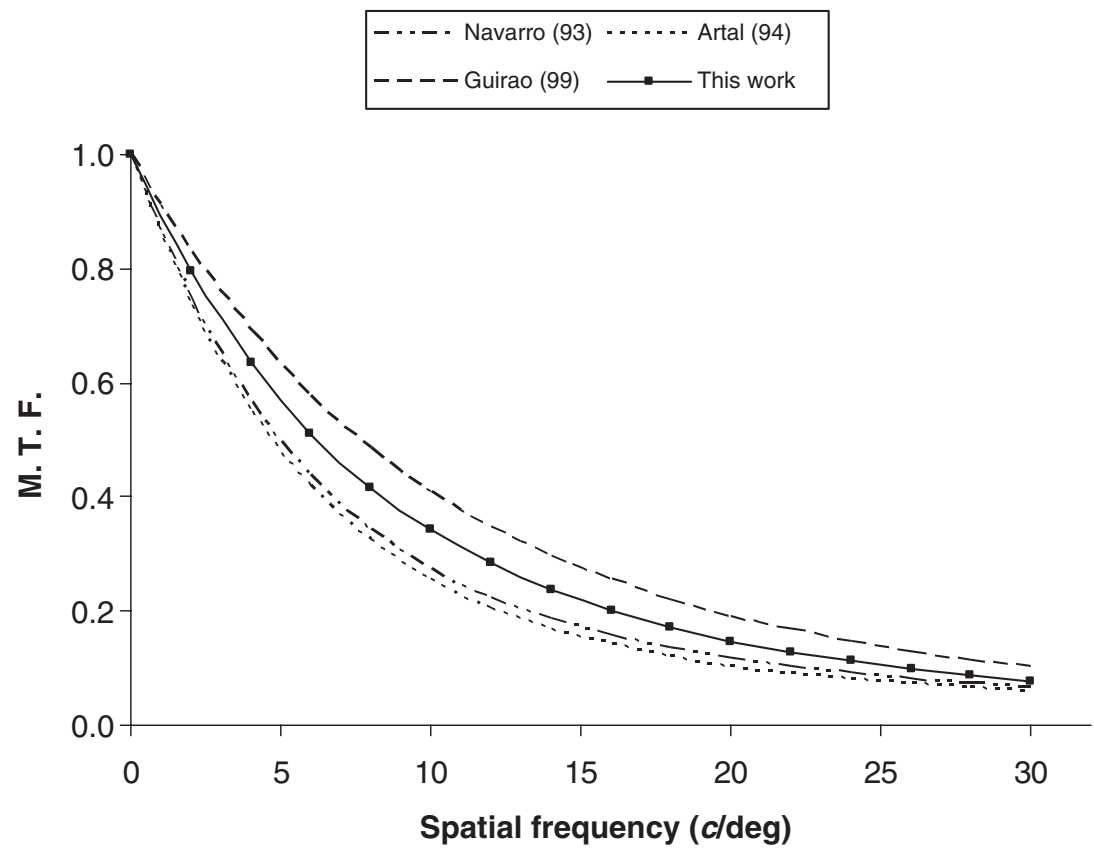

Figure 2. Comparison between the radial MTF here obtained and data reproduced from other workers [29-33] for a pupil diameter of $4 \mathrm{~mm}$.

Table 1. Adjusted parameters for the analytical MTF in equation (21) for a pupil diameter of $4 \mathrm{~mm}$ : comparative results.

\begin{tabular}{lccc}
\hline & \multicolumn{3}{c}{$M T(f)=A \exp (-(f / B))+(1-A) \exp (-(f / C))$} \\
\cline { 2 - 4 } Reference & $\mathrm{A}$ & $\mathrm{B}\left(\mathrm{c} \mathrm{deg}^{-1}\right)$ & $\mathrm{C}\left(\mathrm{c} \mathrm{deg}^{-1}\right)$ \\
\hline$[30]$ & 0.20 & 26.02 & 5.68 \\
{$[32]$} & 0.18 & 25.01 & 5.56 \\
{$[33]$} & 0.25 & 25.42 & 7.81 \\
This work & 0.22 & 24.91 & 7.20 \\
\hline
\end{tabular}

Note: $\mathrm{c} \mathrm{deg}^{-1}$ : cyclon per degree.

proposed by Artal and Navarro [32]. The adjustment parameter $C$ is an estimate of the width in the MTF curve and can thus be regarded as a global index of image quality, similar to the Strehl ratio. The parameter $B$ is related to image quality at medium to high spatial frequencies, but it has no direct significance, in contrast with parameter $C$. Finally, $A$ is a dimensionless free parameter that takes into account the modulation response at high frequencies.

Several researchers have obtained similar values for these parameters [30, 32, 33]. In table 1 we show their results with our own adjustment. Graphically, these results are presented in figure 3. Good agreement between our results and existing data is observable there.

Other clinical parameters have been studied in [34] and good agreement with experimental results is obtained. In this reference, a complete statistical study was 


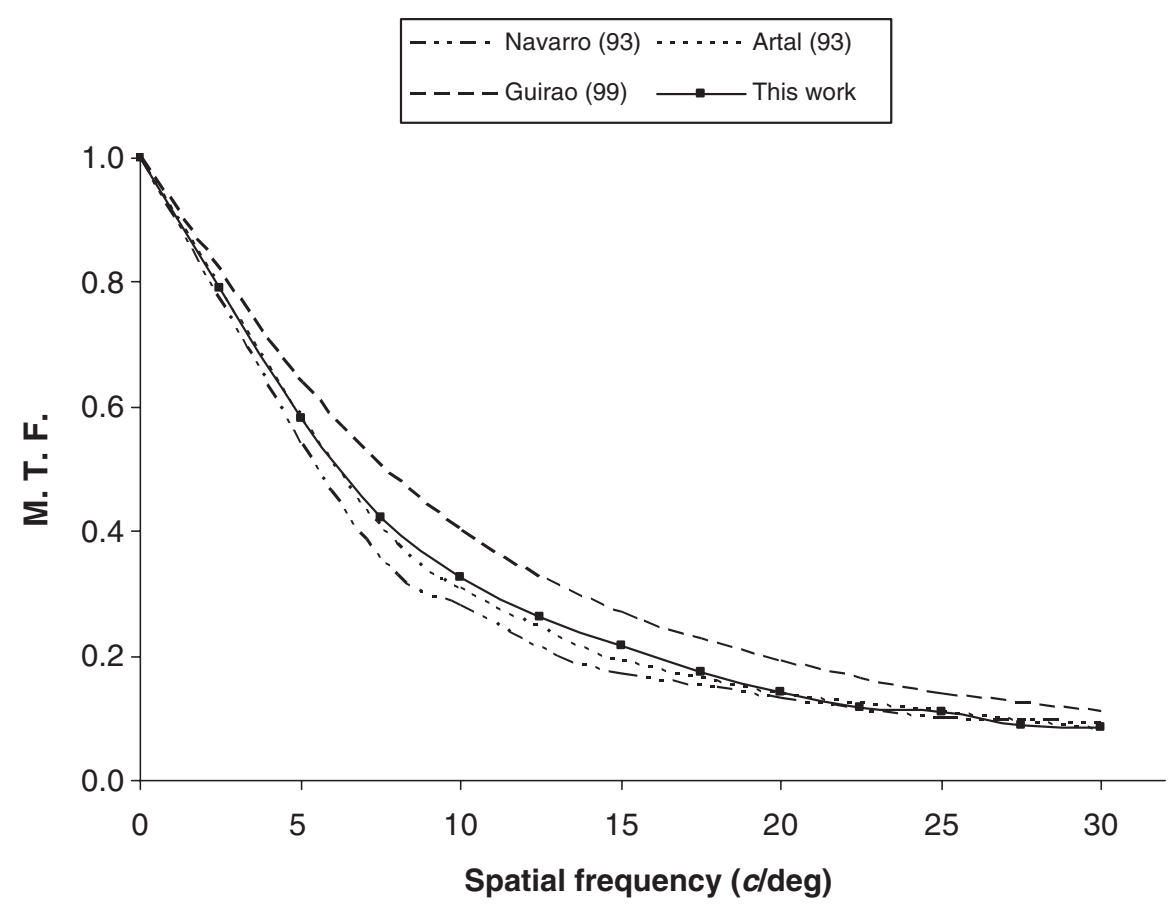

Figure 3. Comparison between the analytical MTF here obtained and analytical representations from other works [30, 32, 33] for a pupil diameter of $4 \mathrm{~mm}$.

made by analysing MTF dependence on the age, pupil size and influence of the Stiles-Crawford pupil for two groups of subjects.

Validation of this technique not only requires only statistical agreement with average results but also must be capable of performing individual predictions. We have measured the refraction of each subject, and its eye total axial length. The algorithm allows exploring a range of distances around the retina and determining the plane with the maximum Strehl ratio. The difference between obtained distance and the retinal position provides the predicted ametropia. Cylindrical predictions were made by comparing distances where the Strehl ratio reaches local maxima with the measured astigmatism. Prediction results are of $0.25 \pm 0.75$ dioptres (expected value \pm standard deviation) for spherical measures and $0.32 \pm 0.33$ dioptres for cylindrical measures. Results are similar to those in [33].

The results which have been presented allow validation of the technique and lead the authors to propose new applications of the model. One of the present authors is checking the performance of a crystalline lens model. In fact, we can change the third step in the algorithm (see section 3.3) and substitute the transmittance of the Kasprzak crystalline lens by any other crystalline lens or even an IOL.

\section{Applications}

The problem of considering a crystalline lens model is that it does not fit the whole population and the number of exceptions is significant. Thus, validation of a new 


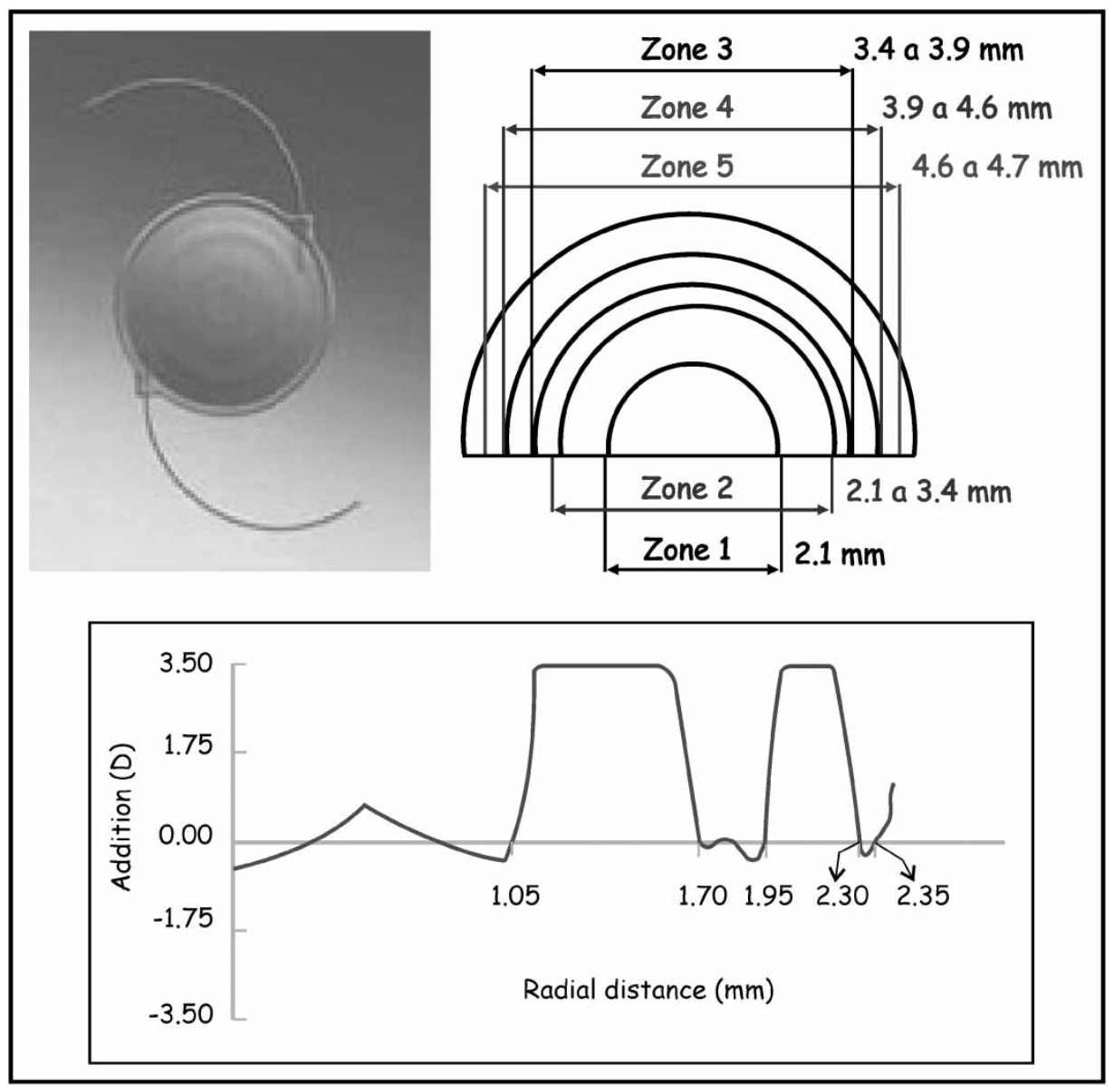

Figure 4. Design and schematic structure of a bifocal IOL AMO ARRAY manufactured by $3 \mathrm{M}$ (D, dioptres).

crystalline model can be made only in a statistical manner. On the other hand, simulation of an IOL can be made specifically for a particular eye. As far as the situation inside the eye, tilt and decentration can be analytically expressed; those data can be incorporated into the general algorithm. Thus, from a particular cornea and a particular IOL, we can generate propagated patterns at any distance inside the eye in a wide variety of situations.

We now give an example of the application of the technique to the study of IOLs. We have studied two different elements: one standard monofocal lens and one multifocal lens. Specifically, we have studied the AMO ARRAY from 3M. This lens is formed by concentric rings which alternate addition powers for far- and near-field vision (figure 4). The result is a lens with a long and very narrow caustic. In figure 5 we show such a caustic for one meridian of an implanted AMO ARRAY obtained for a parallel incoming beam. Note that light concentration is produced in two zones: one corresponding to the near and one to the far addition zones. Note also the 


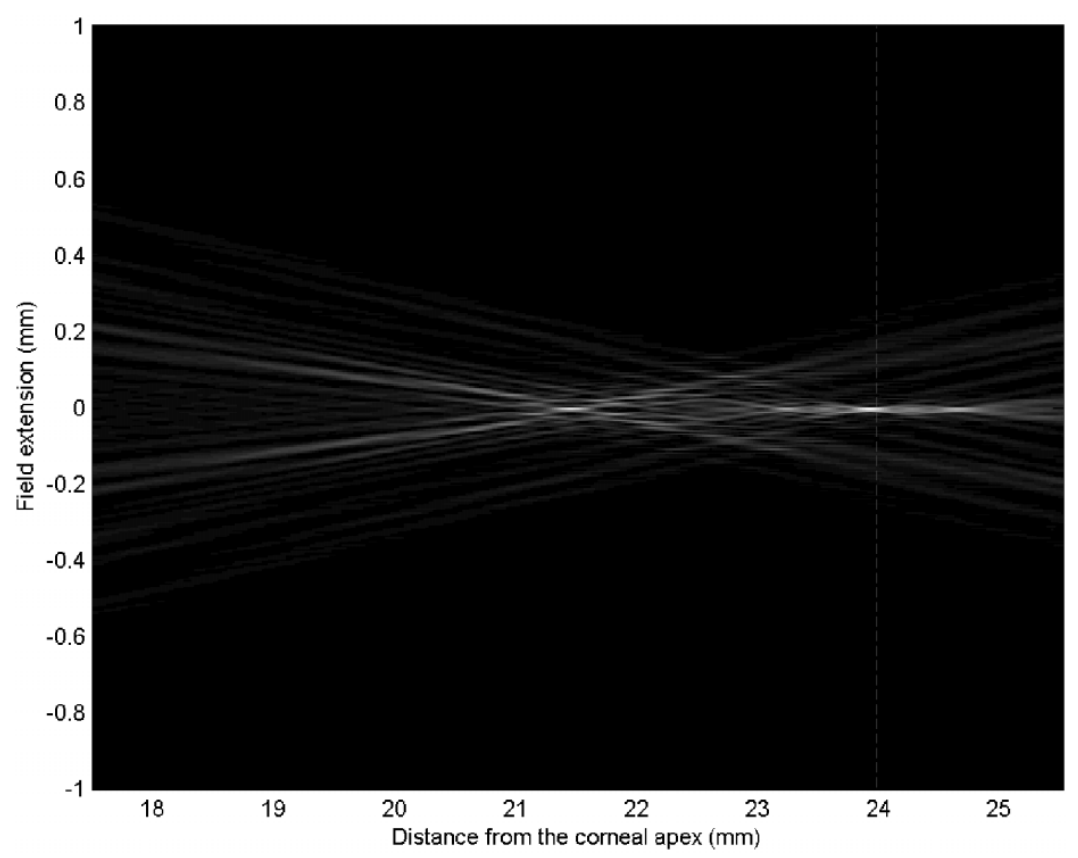

Figure 5. Caustic calculated inside one eye with an implanted AMO ARRAY obtained for a far punctual object. Data are obtained for a pupil diameter of $4 \mathrm{~mm}$. The broken white line indicates the position of the retinal plane.

presence of a focusing zone of some millimetres long. This effect allows the subject to have correct vision in a range of distances.

With our method we have calculated the light pattern at the retinal plane for one aphakic eye in two different situations. First, we have simulated a monofocal intraocular lens. A spot light has been situated at several distances between the remote and the near fixation point. The Strehl ratio is obtained at the retinal plane for all these situations (figure 6). We can see that a maximum is obtained for an object at infinity, while there is a fast decay when approaching the spot. The second situation corresponds to an AMO ARRAY lens. For the object at infinity, the Strehl ratio presents a local maximum which is lower than the maximum obtained in the monofocal case. It should be noted that the decay is not as fast as in the previous case. It is also important to note that there is a second maximum for objects at approximately $80 \mathrm{~cm}$ from the subject. The interval between the two maxima is the region where focused vision is allowed. In figure 7 we present a complementary graphic where the entropy of the image is represented. As explained in [14], the entropy is a measure of the energy dispersion of the image plane. The monofocal lens provides a narrow peak with low energy dispersion that increases as the object approaches. Note that the AMO ARRAY provides a plane zone where dispersion is higher than in the previous case. For objects nearer than $80 \mathrm{~cm}$, dispersion increases and vision becomes blurred. Clinical studies on real patients confirm these situations and state that vision in the accommodation zone is affected by haloes and glares but is good enough to permit reasonable quality of vision. 


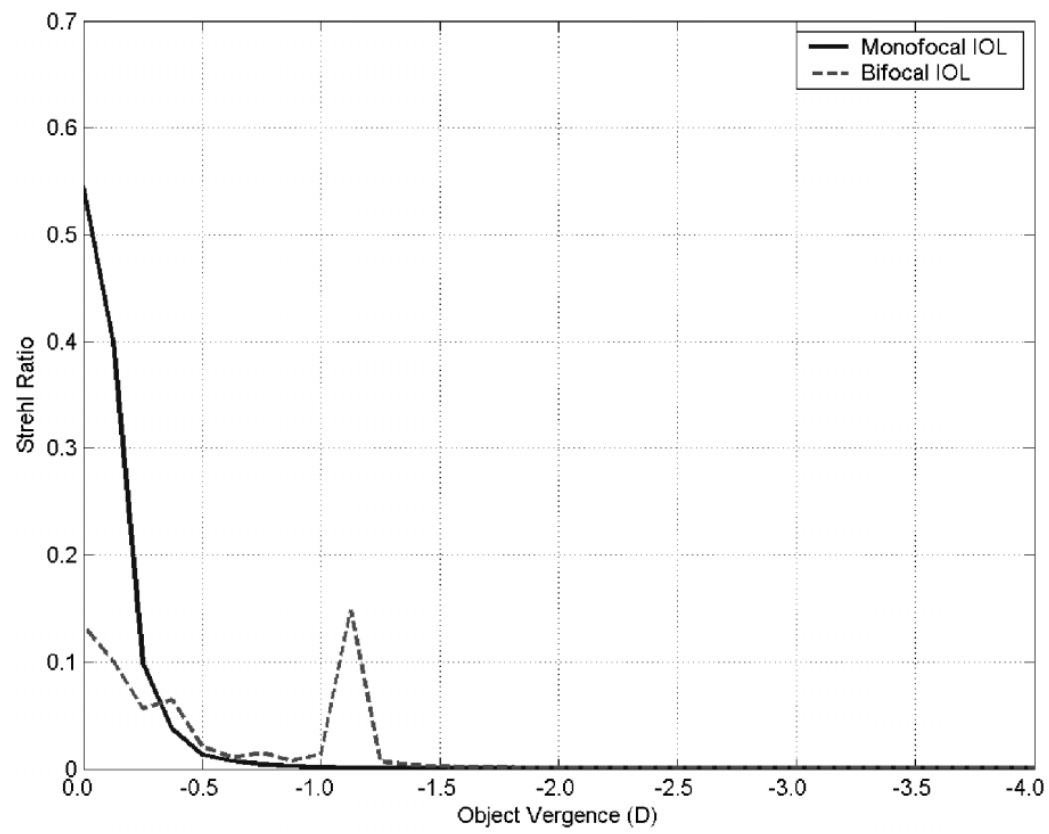

Figure 6. Variation in the Strehl ratio at the retinal plane for different object positions calculated on the retinal plane. Data are obtained for a real eye with a spherical cornea with a pupil diameter of $4 \mathrm{~mm}$. For the simulation we have substituted two different IOLs for the crystalline lens.

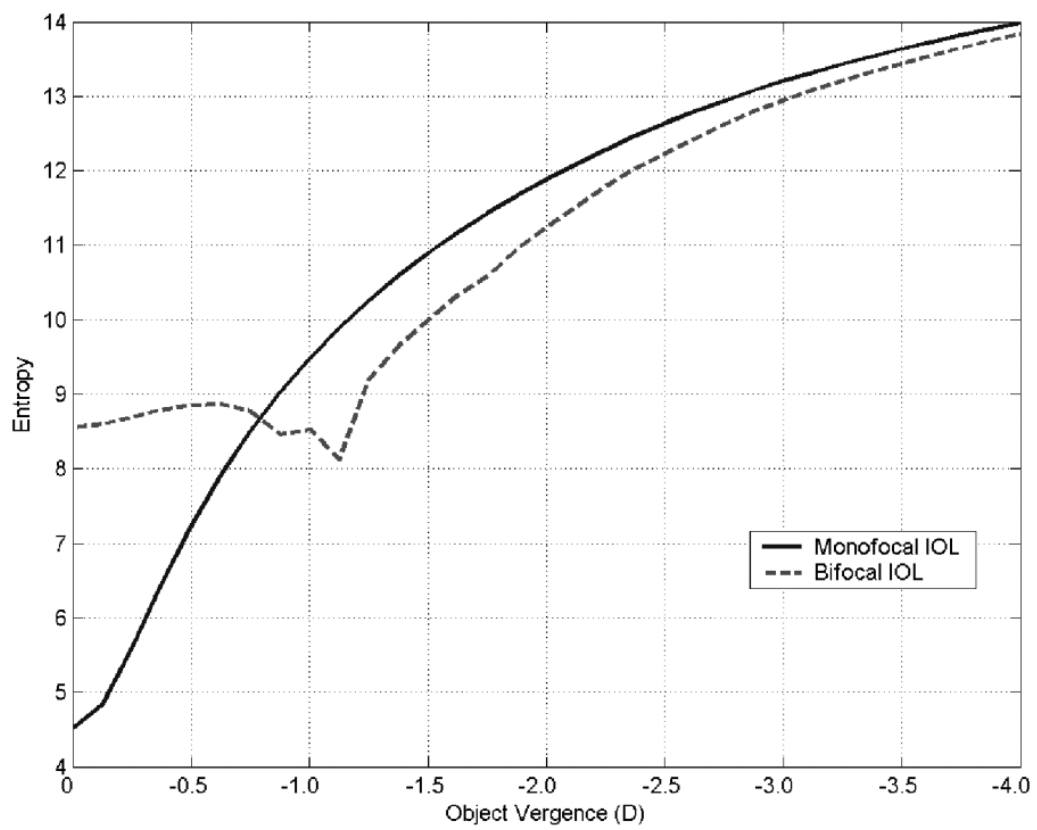

Figure 7. Variation in the entropy at the retinal plane for different object positions calculated on the retinal plane (D, dioptres). Data are obtained for a real eye with a spherical cornea with a pupil diameter of $4 \mathrm{~mm}$. For the simulation we have substituted two different IOLs for the crystalline lens. 


\section{Conclusions}

We have described a complete algorithm to calculate light patterns inside the different ocular media. The full algorithm consists of numerical evaluation of the transmittance of cornea and crystalline lens and the application of a modified spectrum propagation method to obtain light pattern distributions inside the anterior or vitreous chambers.

The method requires considering sampling relations in the propagation kernel, the convergence factors of both cornea and lens, and interference between them. Having in mind all these contour conditions, we have obtained the optimum number of samples that are needed to implement the algorithm.

Under those conditions we have used our method to analyse several parameters of interest in the study of the human eye. We have obtained a typical MTF curve that is successfully compared with similar results from other researchers. The method also allows reasonable predictions to be made on spherical and cylindrical ametropia.

We have also presented some applications of the technique to the study of IOL adaptation. We have obtained the real adaptation range of a commercial multifocal IOL on a real eye, and we have obtained several parameters that may be studied for a complete adaptation of such lenses.

Future research by the present authors is focussed on the implementation of new models of crystalline lenses [35] and IOLs. Data of decentration, tilt or misalignment of the IOL can be also checked and incorporated into future versions of our algorithm.

\section{Acknowledgments}

This work has been partially supported by the Conselleria de Cultura, Educació i Esport of the Generalitat Valenciana, through project GV04A/578.

\section{References}

[1] R. Navarro, J. Santamaría and J. Bescós, J. Opt. Soc. Am. A 21273 (1985).

[2] L.H. Al-Ahdali and M.A. El-Messiery, Appl. Optics 345738 (1995).

[3] G. Smith and D. Atchison, J. Opt. Soc. Am. A. 142537 (1997).

[4] A.M. Pons, A. Lorente, C. Illueca, et al., J. Mod. Optics 461043 (1999).

[5] A.M. Pons, A. Lorente, C. Illueca, et al., J. Mod. Optics 471339 (2000).

[6] C. Illueca, D. Mas, J. Pérez, et al., J. Mod. Optics 48811 (2001).

[7] J. Liang, B. Grimm, S. Goelz, et al., J. Opt. Soc. Am. A 111949 (1994).

[8] J. Liang and D.R. Williams, J. Opt. Soc. Am. A 142873 (1997).

[9] R. Navarro, E. Moreno-Barriuso and C. Dorronsoro, J. Opt. Soc. Am. A 152522 (1998).

[10] S. Marcos, L. Díaz-Santana, L. Llorente, et al., J. Opt. Soc. Am. A 191063 (2002).

[11] J. Santamaría, P. Artal and J. Béscos, J. Opt. Soc. Am. A 41109 (1987).

[12] P. Artal, S. Marcos, R. Navarro, et al., J. Opt. Soc. Am. A 12195 (1995).

[13] S. Marcos, E. Moreno-Barriuso and R. Navarro, Vision Res. 392039 (1999).

[14] D. Mas, J. Pérez, C. Vázquez, et al., J. Mod. Optics 501335 (2003).

[15] D. Mas, J. Pérez, C. Hernández, et al., Optics Commun. 227245 (2003).

[16] G. Smith and D.A. Atchison, Ophthalmic Physiol. Optics 21317 (2001).

[17] H.T. Kasprzak, Ophthalmic Physiol. Optics 2031 (2000).

[18] J.F. Koretz, P.L. Kaufman, N.W. Neider, et al., Vision Res. 291685 (1989).

[19] N. Brown, Expl. Eye Res. 19175 (1975). 
[20] C.A. Cook, J.F. Koretz, A. Pfahnl, et al., Vision Res. 342945 (1995).

[21] W.H. Southwell, J. Opt. Soc. Am. A 17 (1981).

[22] D. Mendlovic, Z. Zalevsky and N. Konforti, J. Mod. Optics. 44407 (1997).

[23] D. Mas, J. García, C. Ferreira, et al., Opt. Commun. 164233 (1999).

[24] D. Mas, C. Ferreira, J. García, et al., Opt. Engng. 391427 (2000).

[25] J.W. Goodman, Introduction to Fourier Optics (McGraw-Hill, New York, 1968), p. 78.

[26] A. Guirao and P. Artal, J. Opt. Soc. Am. A 17955 (2000).

[27] A. Popiolek and H.A. Kasprzak, Optom. Vis. Sci. 76720 (1999).

[28] J.D. Marsack, L.N. Thibos and R.A. Applegate, J. Vision 4322 (2004).

[29] P. Artal, M. Ferro, I. Miranda, et al., J. Opt. Soc. Am. A 101656 (1993).

[30] R. Navarro, M. Ferro, P. Artal, et al., Appl. Optics 326359 (1993).

[31] P. Artal, S. Marcos, I. Miranda, et al., Opt. Engng. 34772 (1995).

[32] P. Artal and R. Navarro, J. Opt. Soc. Am. A 11246 (1994).

[33] A. Guirao, C. González, M. Redondo, et al., Invest. Ophthalmol. Vis. Sci. 40203 (1999).

[34] J. Pérez, D. Mas, J.J. Miret, et al., Optik (submitted) (2004).

[35] D. Siedlecki, H. Kasprzak and B.K. Pierscionek, Optics Lett. 291197 (2004). 